\title{
BioéthiqueOnline
}

\section{La puissance technique de la médecine : des pistes de réflexion}

\section{Andrée Duplantie}

Volume 5, 2016

URI : https://id.erudit.org/iderudit/1044269ar

DOI : https://doi.org/10.7202/1044269ar

Aller au sommaire du numéro

Éditeur(s)

BioéthiqueOnline

ISSN

1923-2799 (numérique)

Découvrir la revue

Citer ce compte rendu

Duplantie, A. (2016). Compte rendu de [La puissance technique de la médecine : des pistes de réflexion]. BioéthiqueOnline, 5. https://doi.org/10.7202/1044269ar
Résumé de l'article

Dans son livre Réflexion éthique et pratiques soignantes - Points de repère, Christian Gilioli relate l'évolution de la médecine et de l'éthique lesquelles entraînent des questionnements de natures philosophique, scientifique, technologique, sociale, économique et politique. Il propose des pistes de réflexions touchant la complexité de l'éthique contemporaine, dans un monde moderne toujours à la recherche du bonheur et de la santé et refusant le vieillissement et la souffrance. 


\title{
La puissance technique de la médecine : des pistes de réflexion
}

\author{
COMPTE RENDU / REVIEW
}

Andrée Duplantie, Inf., M.Sc., M.A. bioéthique ${ }^{1}$

$$
\text { Reçu/Received: } 14 \text { Mar } 2016 \quad \text { Publié/Published: } 18 \text { May } 2016
$$

Éditrices/Editors: Lise Lévesque \& Mariana Nunez

2016 A Duplantie, Creative Commons Attribution 4.0 International License

\section{Résumé}

Dans son livre Réflexion éthique et pratiques soignantes Points de repère, Christian Gilioli relate l'évolution de la médecine et de l'éthique lesquelles entraînent des questionnements de natures philosophique, scientifique, technologique, sociale, économique et politique. II propose des pistes de réflexions touchant la complexité de l'éthique contemporaine, dans un monde moderne toujours à la recherche du bonheur et de la santé et refusant le vieillissement et la souffrance.

\section{Mots clés}

éthique contemporaine, pratiques soignantes, évolution de la médecine, personne, développement technologique

\section{Summary}

In his book, Réflexion éthique et pratiques soignantes Points de repère (Ethical reflection and care practices Landmarks), Christian Gilioli describes the evolution of medicine and ethics the associated questions of a philosophical, scientific, technological, social, economic and political nature. He proposes further reflections pertaining to the complexity of contemporary ethics in the modern world continuously in search of happiness and health, and in denial of ageing and suffering.

\section{Keywords}

contemporary ethics, care practices, evolution of medicine, person, technological development

Affiliations des auteurs / Author Affiliations

${ }^{1}$ Experte conseil en éthique clinique, Montréal

\section{Correspondance / Correspondence}

Andrée Duplantie, aduplantie@bioethique.ca

Conflit d'intérêts

Aucun déclaré

\section{Conflicts of Interest}

None to declare

\section{Introduction}

Le livre, Réflexion éthique et pratiques soignantes - Points de repère [1], aborde la question de l'éthique et de la santé telle qu'elle se pose aujourd'hui dans le domaine de la santé. L'auteur, Christian Gilioli est cadre supérieur de santé et actuellement responsable des droits du patient, des associations et des cultes à la direction qualité des hôpitaux universitaires Henri-Mondor. II enseigne depuis de nombreuses années l'éthique soignante en formation universitaire, Université de Paris-Est Créteil. Le contenu de son livre est vaste, dense et riche de données, de questionnements et de réflexions.

La première partie, la plus constitutive de cet ouvrage, trace l'évolution de l'éthique, de la médecine et de la santé. La deuxième partie aborde divers concepts et questionnements dont ceux liés à la maladie, à la démarche éthique, aux problèmes éthiques contemporains, à la médecine et la technologie ainsi que ceux liés à la personne et à l'euthanasie. Gilioli puise à divers domaines et cite de nombreux auteurs, dont les principaux sont présentés avec un court résumé de leurs œuvres, en début de livre (p.17-22).

La préface de ce livre, signé par Dominique Folscheid, philosophe français et spécialiste des questions d'éthique du champ médical, pose d'entrée de jeu la question la plus importante et la plus prégnante de cet ouvrage sur l'éthique et les pratiques soignantes: "Pourquoi notre médecine moderne, devenue si savante et si efficace depuis quelques décennies, nous pose-t-elle tant de problèmes? "C'est un peu à cette question que tente de répondre Gilioli. 


\section{Transformation des pratiques soignantes}

L'auteur met en contexte la médicalisation de la vie dans notre monde occidental de même que l'extraordinaire développement des connaissances scientifiques et médicales, et d'une technologie de plus en plus perfectionnée et coûteuse.

L'auteur nous propose « d'effectuer un survol panoramique de l'histoire de la médecine et, surtout, de tenter d'approcher le concept de maladie dans son évolution historique » (p.84), depuis Hippocrate, Galien, Averroès de même que la théorie des humeurs, l'épidémie et le phénomène de la contagion. Passant rapidement à l'idée de la santé dans un monde moderne, l'auteur passe également du préventif au prédictif puis à la génétique d'aujourd'hui. Ainsi, notre société est toujours à la recherche d'un monde meilleur où la souffrance est supprimée et la vie prolongée. Tout cela au prix de coûts terribles pour des systèmes de santé au bord de l'implosion, occasionnant notamment des problèmes liés à l'accès équitable aux soins. Cette situation exige une réflexion éthique poussée. Les problèmes éthiques dans les pratiques médicales et soignantes sont spécifiques et uniques, nécessitant un débat d'idées et d'appréhension des problèmes et enjeux soulevés.

Abordant la démarche éthique face à des problèmes très contemporains, comme la réanimation, la non-consubstantialité de l'éthique et de la médecine, la procréatique et le progrès de la médecine, etc. Gilioli nous met en garde de ne pas tomber dans un relativisme des valeurs pour justifier notre point de vue. II insiste sur les différences entre l'approche anglo-saxonne (modèle autonomique) très largement organisée sur le mode d'une éthique contractuelle et l'approche française (modèle basé sur le paternalisme). II précise (p.113) :

Ces deux modèles s'opposent :

- le modèle français dominé par la poursuite du bien, c'est-à-dire supporté par le principe de bienfaisance;

- le modèle anglo-saxon marqué par le respect inconditionnel de la liberté du malade au risque même de l'indifférence.

Ces modèles présentent tous deux des imperfections dont l'effet d'aliénation dans le modèle paternaliste et le désinvestissement du médecin dans le modèle basé sur l'autonomie du patient. De cette constatation, une voie médiane se retrouve dans la position de l'auteur Anne Fagot-Largeault. Cette position fait appel à un « paternalisme tempéré » qui exclurait la subordination paternaliste et la froide indifférence de l'autonomie (p.114), voie rendue très complexe par la judiciarisation des rapports médecins-malades selon Gilioli.

Dans le but d'un agir juste, la démarche éthique est vue comme un processus d'intellectualisation de ce que nous observons et comprenons du réel. Déclinée en sept étapes, la réflexion éthique conduisant à la prise de décision est celle de Monique Canto-Sperber [2, p.99]. Cette dernière considère que cette démarche s'organise avant tout sur la raison, sur la rationalité intellectuelle, même si l'on sait que la décision repose en partie sur les émotions, les affects dont il faut se méfier.

\section{La personne: sujet ou objet}

La question de la personne apparaît centrale à la réflexion éthique telle que soulevée par l'auteur. Ce qui domine dans la conception moderne, c'est " la conscience morale - c'est-à-dire la conscience d'être au monde -, élément déterminant pour qualifier un être humain de personne. " (p.145) Mais, reste que la discussion de plusieurs auteurs autour de cette notion de la personne ne fait pas consensus. En effet, ce ne sont pas tous les auteurs qui considèrent la personne sous le même angle. En France, remarque Gilioli, « nous pensons par exemple que tous les êtres humains sont des personnes, ce que contestent certains penseurs américains en particulier. ॥ (p.98) Se poser la question de la personne aujourd'hui n'est donc pas superflu pour stimuler la réflexion éthique, face à 
une médecine de plus en plus technicisée impliquant la personne (le patient) souvent prise et traitée comme objet plutôt que sujet. La question de la réanimation, où les équipes soignantes sont confrontées continuellement au choix de la poursuite ou non de la technique comme traitement (personne objet), au point de perdre de vue l'objectif de soin (personne sujet).

\section{La volonté de maîtrise}

La question de la personne soulève forcément celle de la volonté de maîtrise du sujet moderne à la recherche de ce monde meilleur. La réflexion éthique de Gilioli autour de la technicisation de la réanimation illustre la complexité de ces trois pôles : éthique, nature et technique. Par exemple, la réflexion éthique est davantage aigue et centrale en réanimation (p.124), et nous pouvons dire en réanimation néonatale, et auprès de tant d'autres patients de tous âges et de toutes conditions de santé. Ainsi, poursuit l'auteur, "les résultats parfois catastrophiques de réanimation sont comparables aux effets qu'un développement industriel non maîtrisé peut avoir sur la nature. » (p.126)

Bien que Gilioli n'apporte pas d'exemples concrets, nous pouvons aisément dire que la médecine, toujours portée de plus en plus vers le progrès et le développement incessant de nouvelles technologies, peut dans certaines situations promouvoir à tout prix, la viabilité des nouveau-nés ou des personnes plus âgées, au risque de prolonger leur vie sans ou sinon avec si peu de qualité de vie. Les exemples sont nombreux dans le milieu de la santé et dans les écrits scientifiques. Cependant, Gilioli ajoute qu'il ne s'agit pas de dénoncer les actions médicales proprement dites mais plutôt de « repérer les dérives d'une modernité marquée par l'apparition de possibilités techniques extrêmement puissantes. " (p.126) À cet effet, il semble intéressant pour Gilioli de mentionner (p.131) la position du philosophe Michel Onfray [3] qui croit plutôt que ces craintes ne sont que le reflet d'une morale judéo-chrétienne et qu'il serait temps de se tourner vers une éthique délaissée de ces valeurs religieuses.

D'ailleurs, ces dérives ne seraient pas issues uniquement des nouvelles possibilités techniques, mais surtout de la volonté de maîtrise totale de la maladie et de la santé. En effet, insiste Gilioli, le désir de tout contrôler est très présent chez l'homme moderne. Et il semble toujours obligatoire de trouver un responsable, voire un coupable, " comme s'il était possible de construire un monde où tout danger serait aboli. » (p.151).

S'il n'est pas nouveau de voir l'homme vouloir tout contrôler, son désir de ne pas souffrir est caractéristique de l'homme moderne. D'où les débats sur l'aide au mourir dans la dignité, comme nous pouvons l'observer en France [4], au Québec [5] ou ailleurs. La demande d'euthanasie, serait l'exacte manifestation de l'homme moderne voulant tout gérer, de sa naissance à sa mort. Par ailleurs, en France, la loi continue d'interdire toute forme d'euthanasie et de suicide assisté, mais encourage la sédation de la souffrance par tous les moyens [6]. Ce même chapitre termine par une discussion sur la dignité humaine. Citant Jacques Ricot [7] " La dignité dans l'usage contemporain, désigne cette sorte d'élégance qui épargne à autrui le spectacle de nos propres maux. ॥ (p.164) Mais Gilioli poursuit en spécifiant que la notion même de dignité touche l'ensemble des êtres humains puisque ces derniers sont dignes, simplement du fait qu'ils sont des humains.

\section{Conclusion}

Ce livre mérite d'être lu et relu car il y a matière à réflexion et à discussion. Le contenu est condensé, puisant à de nombreuses références, souvent philosophiques et abordant la réflexion éthique dans le domaine de la santé. II s'adresse en principe à tous ceux et celles qui s'intéressent aux questions d'éthique soulevées par la technicisation de la médecine, et qui désirent replonger dans les précieuses réflexions des philosophes comme Ricœur, Bourdieu, Levinas, Foucault, Gadamer et tant d'autres grands penseurs nécessaires à la profondeur de nos propres réflexions. Les professionnels 
de la santé attirés par le titre pourraient rester sur leur appétit ou bien se sentir déroutés du fait que ce livre aborde peu ou pas directement les pratiques soignantes ou de manière différente de celle de la littérature nord-américaine. Par ailleurs, les problèmes éthiques abordés dans son livre sont universels, mais les tentatives de réponses concrètes qu'il évoque se rapportent surtout à diverses situations ou instances en France, dont le reconnu CCNE (Comité Consultatif National d'Éthique).

Enfin, il est à propos de se demander pourquoi notre médecine moderne scientifique et de plus en plus technologique, engendre autant de questionnements, de problèmes et de soucis actuellement? S'il n'existe pas de réponse à cette grande question posée, qu'à cela ne tienne! Car vous trouverez dans ce livre des éléments de réponses et de réflexions philosophiques ainsi que certaines pistes d'actions ancrées dans des pratiques soignantes actuelles comme le réaménagement du lien médecin-patient ou soignant-patient.

\section{Références}

1. Gilioli C. Réflexion éthique et pratiques soignantes - Points de repères. Paris, France : Éditions Lamarre ; 2015. 171 pages.

2. Canto-Sperber M. L'inquiétude morale et la vie humaine. Paris : Presses universitaires de France ; 2001, 293 p.

3. Onfray M. Fééries anatomiques. Paris : Éditions Grasset ; 2003, 352 p.

4. Comité Consultatif National d'Éthique. Avis 26,58 et 63 sur Fin de vie, autonomie de la personne, volonté de mourir. Paris : 1991, 1998 et 2000.

5. Assemblée Nationale du Québec. Loi concernant les soins de fin de vie. Loi sanctionnée le 10 décembre 2015. Québec.

6. Comité Consultatif National d'Éthique. Avis 121 Fin de vie, autonomie de la personne, volonté de mourir. Paris : 2013.

7. Ricot J. Dignité et euthanasie. Nantes : Plein Feux ; 2003.

\section{Lectures recommandées}

1. Bourdieu P. Leçon sur la leçon. Paris : Éditions de Minuit ; 1982, 60 p.

2. Fagot-Largeault A. Médecine et philosophie. Paris : Presses universitaires de France ; 2010, $288 \mathrm{p}$.

3. Foucault, Michel. Surveiller et punir. Paris : Gallimard ; 1993, 400 p.

4. Gadamer H G. Philosophie de la santé. Paris : Éditions Grasset ; 1998.

5. Levinas E. Totalité et infini. Essai sur l'extériorité. Paris : Le livre de poche ; 2006, 352 p.

6. Levinas E. Éthique et infini. Paris : Fayard ; 1982, 144 p.

7. Ricœur P. Soi-même comme un autre. Paris : Éditions du Seuil ; 1996, 448 p. 